\title{
Efectos de corto plazo de la inmigración venezolana en el Perúi
}

\section{Short-term effects of Venezuelan immigration in Peru}

\section{RESUMEN}

Jhon Valdiglesias Oviedo ${ }^{2}$

El artículo reafirma los efectos positivos de la inmigración en las economías de destino en el largo plazo, sin embargo, pretende explicar los problemas que se presentan en el corto plazo, a propósito de la reciente inmigración venezolana en el Perú. Estos problemas se derivan de las características de la economía peruana como la presencia de un amplio sector informal o el déficit de capital físico e infraestructura pública. Asimismo, la duración de estos problemas dependerá de la calidad de las políticas públicas que se implementen para alcanzar los beneficios de la inmigración. Finalmente, se propone algunas recomendaciones de políticas.

Palabras clave: Inmigración; empleo; informalidad.

JEL: J01, E26

\section{ABSTRACT}

The article reaffirms the positive effects of immigration in the long term, however, with the intention of emphasizing the problems that arise in the short term, regarding the recent Venezuelan immigration in Peru. These problems derive due to the characteristics of the economy, as well as the nature of a large informal sector or the deficit

1 El presente artículo se desprende de una serie de estudios de investigación sobre el impacto de la inmigración en el Perú encargadas por el Estado peruano.

2 El autor es economista de la Universidad Nacional Mayor de San Marcos en Perú. Asimismo, realizo estudios de maestría en la National Chengchi University de la República de China (Taiwán). Posteriormente realizo un doctorado en economía en la University of International Business and Economics de la República Popular de China

(C) Los autores. Este artículo es publicado por Pensamiento Crítico de la Facultad de Ciencias Económicas, Universidad Nacional Mayor de San Marcos. Este es un artículo de acceso abierto, distribuido bajo los términos de la licencia Creative Commons Atribucion - No Comercia_Compartir Igual 4.0 Internacional. (http://creativecommons.org/licenses/by-nc-sa/4.0/) que permite el uso no comercial, distribución y reproducción en cualquier medio, siempre que la obra original sea debidamente citada. 
of physical capital and public infrastructure. It is worth to note that the duration of these problems will depend on the quality of the public policies that will be implemented to achieve the benefits of immigration. Finally, some policy recommendations are proposed.

Keywords: Immigration; employment; informality.

JEL: J01, E26

\section{Introducción}

Países como Estados Unidos, Alemania, Canadá o Australia han beneficiadas sus economías gracias a los flujos inmigratorios. El impacto ha sido mayor cuando los inmigrantes cuentan con un alto nivel educativo o de capital humano. Es así que, la historia económica del Perú también registra efectos positivos de la inmigración; por ejemplo, los flujos extranjeros de italianos, chinos y japoneses. Es importante destacar que los efectos positivos se lograron en el largo plazo.

En la figura 1 se muestra un equilibrio inicial en el punto " $A$ " en el mercado laboral. Como resultado del flujo inmigratorio, la curva de oferta de trabajo se desplaza a la derecha, generando un nuevo equilibrio en el punto "B"; lo que origina una caída en los salarios. Sin embargo, la teoría predice un aumento de la demanda de trabajo debido a que las empresas deciden incrementar su producción ante el mayor número de consumidores. Como resultado se tiene un nuevo equilibrio en el punto ' $C$ ', donde aumenta el salario, el empleo y el tamaño de la economía.

La teoría establece que el principal efecto de la inmigración sobre el mercado laboral, en el corto plazo, recae en la caída de los salarios, ello debido a la existencia de más personas en búsqueda de empleo, siguiendo el criterio de la ley de la oferta y la demanda. De esta manera, la inmigración se entiende como un choque de mayor oferta dentro del mercado laboral. En principio, la caída de los salarios sería para todos los que participan en este mercado; es decir, tanto para los extranjeros como para los trabajadores locales.

Es importante destacar, a partir de la literatura revisada sobre inmigración, que entre las características más destacadas que presentan, en promedio, los inmigrantes; es que estos tienden a ser más jóvenes, y también a contar con menor educación formal respecto a los trabajadores locales. 


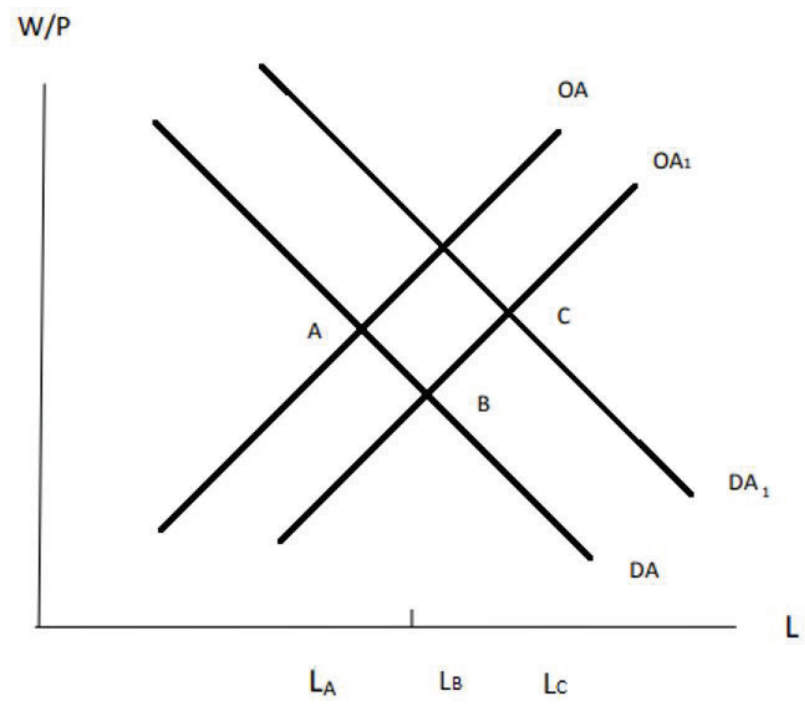

Figura 1. Efecto de la inmigración en el mercado de trabajo

Fuente: Elaboración propia

Si bien se señaló un choque de oferta laboral que hace caer los niveles de salarios, ello sería luego contrarrestado por un choque de la demanda de trabajo a través de aumentos de stocks de capital, por parte de las empresas. De hecho, la teoría sobre inmigración señala que ésta por si misma hace aumentar la demanda de trabajo, contrarrestando los efectos iniciales de la sobreoferta laboral; sin embargo, este efecto sería en el mediano o largo plazo, dependiendo del tipo de economía.

En efecto, existen diferentes efectos en el mercado laboral por causa de la inmigración por cada región o país. Estas diferencias se deben a diferentes contextos institucionales en variables como salario mínimo legal, protección legal al empleo, flexibilidad laboral, etc. Un estudio sobre migración rusa en Israel estimó que tomó entre 4 a 7 años disipar los efectos iniciales en la reducción del salario promedio ${ }^{3}$.

Asimismo, la inmigración es capaz de aumentar las brechas salariales y por tanto la desigualdad. En esa línea, dentro de los grupos más vulnerables, los más amenazados, entre la población local, por efectos de la 
inmigración serían los trabajadores menos calificados. De otro lado, tenemos los inmigrantes que cuentan con legalidad, quienes a su vez poseen mayor nivel educativo respecto a los inmigrantes ilegales.

Las evidencias registran que países de la OCDE como Irlanda, Inglaterra y Suiza se han visto beneficiados por efectos positivos, en términos del crecimiento de sus economías a partir del recibimiento de inmigrantes con alto nivel educativo. Los analistas consideran estos efectos positivos como significativos. De hecho, países como Canadá, Australia, Estados Unidos, Inglaterra, Suiza y Luxemburgo son considerados como los mayores imanes de inmigraciones internacionales, los cuales presentan estos efectos positivos mencionados en el largo plazo. En el caso de los Estados Unidos, no refleja caída en los salarios en el corto plazo; por el contrario, presenta efectos positivos más inmediatos ${ }^{4}$.

Desde 1970, aproximadamente 30 millones de inmigrantes legales habrían llegado a Estados Unidos. Se estimó que, hasta 1960 estos inmigrantes eran predominantemente de Europa, desde esta fecha predominaron los inmigrantes de Asia y América Latina, quienes son menos educados y perciben menores salarios que los trabajadores locales. La evidencia empírica muestra la existencia de diferentes efectos en la economía de ese país, a partir de la inmigración de diferentes grupos.

Un estudio de los efectos de la migración en la economía alemana determinó que existen efectos significativamente positivos en su economía; destacando que la mayoría de inmigrantes presentan, al principio, barreras(como el idioma) que van siendo superados de forma gradual. A partir de estas experiencias, citadas en el presente estudio, se recomienda diseñar políticas migratorias enfocadas en atraer inmigrantes altamente calificados para obtener similares resultados positivos a países como Inglaterra y Estados Unidos ${ }^{5}$.

Actualmente la inmigración es uno de los temas más populares de debate en Inglaterra. A pesar que la opinión pública se manifiesta en contra de este; las cifras de inmigración vienen en aumento considerablemente. En este país, los inmigrantes presentan desventajas en la competencia por

4 Docquier, F., Özden Ç., Peri G. (2011), página 1.

5 Beyer, R. C. M. (2016). 
empleo, respecto a los locales. En un documento se destaca que los trabajadores locales con menor grado de formación, son los que afrontan los mayores efectos de la inmigración. En efecto, la primera generación de inmigrantes mantiene distancia con los nativos en términos de acceso al mercado laboral; distancia que recién se acortaría en la segunda generación, la cual obtiene grandes logros educativos ${ }^{6}$.

Contrariamente al sentido común de la opinión pública, acerca del hecho que los inmigrantes reducen los sueldos y toman el trabajo de los nativos, la literatura académica presenta resultados contrarios. Los académicos afirman que, en el peor de los casos, los efectos negativos serían marginales. De esta manera, se contaría con caídas prácticamente insignificantes en los salarios promedios. Al mismo tiempo, señalan a la educación, la tercerización, los cambios tecnológicos, etc., como los principales determinantes del nivel del salario.

Partiendo de la lógica de que los inmigrantes afectan más a ciertos grupos de trabajadores, queda la tarea de focalizar el apoyo del gobierno central en los grupos más afectados. Esto va a depender de los tipos de trabajo que más concuerden con los nuevos migrantes y que no cuenten con las barreras como el idioma, el conocimiento cultural o experiencia local.

Pero, según los académicos, similar al caso del salario, analizado previamente, los efectos en el empleo también serían marginales. De un lado, muchos trabajadores locales poseen ventajas comparativas en sus puestos de trabajo que los protegen ante una potencial competencia. En muchos casos, no existe sustitución entre los trabajadores locales e inmigrantes. De otro lado, los puestos que concentran a los trabajadores con mejores cualificaciones, que podrían ser afectados (en principio); tampoco serían afectados considerablemente dado que se considera estos sectores como muy flexibles; y por tanto, es factible que el empleador aumente el empleo y, así contratar más trabajadores.

Los escasos casos de reducción en los salarios o el empleo se darían exclusivamente en el corto plazo; y en grupos específicos. Por otro lado, existe un amplio consenso en que en el largo plazo se diluye cualquier de los dos efectos negativos mencionados. En el caso de países europeos

6 Dustmann, C., Hatton, T. and Preston, I. (2005) 
existen resultados disimiles; por ejemplo, para el caso de Alemania los efectos en los salarios son casi nulos; mientras que para otros casos se registran impactos negativos, aunque moderados.

Aunque la mayoría de estudios concluyen con efectos estadísticamente insignificantes en el salario, a partir de aumentos en la participación de los inmigrantes en la PEA, también existen excepciones. En ese marco, se presenta los dos extremos más populares encontrados en la literatura, que se focalizan en estudiar la inmigración en los Estados Unidos. En un extremo tenemos el estudio de David Card quien estima que aumentos en $10 \%$ en la participación de inmigrantes en la fuerza de trabajo aumenta el salario en 6\%; mientras que en el otro extremo tenemos a George Borjas quien encuentra que el mismo aumento de inmigrantes reduce los salarios entre $3 \%$ a $4 \%{ }^{7}$.

La inmigración no afecta a todos los trabajadores locales por igual. Existen sectores en los cuales hay una competencia más directa. Principalmente, los trabajadores de menor calificación son los más afectados por esta mayor competencia. Los estudios confirman que incluso dentro de los grupos de trabajadores con baja calificación existen subgrupos que son fuertemente afectados, mientras más cerca estén de los inmigrantes en términos de competencia.

Otro grupo afectado por los inmigrantes nuevos son los inmigrantes previos; es decir, aquellos que llegaron previamente. Esto se debe a similares características entre estos dos grupos; creando así una fácil sustitución en el trabajo entre estos; sobre todo desde el punto de vista del empleador. En efecto, diversos estudios señalan que los inmigrantes previos serían más vulnerables que los trabajadores locales.

La flexibilidad del mercado laboral puede hacer que la inmigración afecte más al desempleo. Por ejemplo, las diferencias en términos de legislación laboral entre Estados Unidos y Europa hacen que los segundos presenten un mercado laboral menos flexible, y por lo tanto, los efectos de la inmigración se dirigen más directamente hacia el desempleo en lugar de los salarios.

También existe evidencia de una mayor disparidad o desigualdad en el ingreso entre los trabajadores altamente calificados y los poco califica-

7 Somerville, W.; Sumption, M. (2009). 
dos. En efecto, debido a que la mayor competencia se dará en los segundos, dado que competirán con los nuevos migrantes, el salario de este grupo se reducirá. Sin embargo, por defecto, este efecto se considera también como minúsculo en la desigualdad.

Por su parte, los nuevos inmigrantes contribuirán al aumento de la demanda de más bienes y servicios que consumirán. Como consecuencia, se obtendrá una mayor demanda de trabajo por parte de las empresas, mayor eficiencia en el mercado de trabajo y aporte al crecimiento económico de largo plazo. En el largo plazo, también se cuenta con mayor presión a los trabajadores locales en hacerse más competitivos, quienes tendrán que invertir en una mayor capacitación o especialización ${ }^{8}$.

Una pregunta a tener en cuenta es por qué existe tal divergencia entre la opinión pública y los estudios académicos. Los primeros manifiestan un considerable efecto negativo del proceso inmigratorio; mientras que lo segundos todo lo contrario. En todo caso, existe una coincidencia en términos de diseñar políticas en favor de los trabajadores menos calificados o los grupos más vulnerables. En Inglaterra, el sector público contrata un número significativo de inmigrantes tanto directamente, así como a través de sub-contratación ${ }^{9}$.

Los efectos de la inmigración también pueden evaluarse desde una división en dos niveles, según el grado de calificación de los inmigrantes. De esta manera, los académicos han clasificado a los inmigrantes en dos grupos, los bajos y los altamente calificados. Son las migraciones masivas y desautorizadas las que básicamente generan inmigrantes de baja calificación (el primer grupo); asimismo, este grupo es el único que generaría efectos negativos, aunque modestos, en los balances fiscales, así como en los trabajadores locales. En efecto, se espera que los impuestos que estos aportan sean menores a los gastos fiscales que reciben a través de bienes públicos.

De otro lado, los inmigrantes con alta calificación presentan efectos positivos en prácticamente todos los ángulos donde el impacto ha sido analizado; sobre todo ha promovido la innovación y el emprendimiento en los sectores tecnológicos más altos. De otro lado, los inmigrantes con alta

8 Ídem.

9 Ídem. 
calificación ofrecen un superávit fiscal debido a que pagan impuestos en sumas monetarias más altas respecto a los bienes públicos que reciben. Estas evidencias sugieren reformas en la política migratoria en términos de acelerar la legalización de inmigrantes con altos niveles de calificación.

En Estados Unidos, los inmigrantes han impulsado la economía constantemente, fortalecido la productividad, la innovación e incluso mantenido los precios bajos, contrarrestando la inflación y enriqueciendo la cultura del país. Por otro lado, de los efectos negativos, ya se mencionó la presión en el déficit fiscal, el segundo es la desigualdad en la distribución de los beneficios de la inmigración. Respecto a este último punto, los sectores más pobres se benefician mucho menos que los sectores más ricos. De hecho, los de más bajo nivel de calificación son lo que asumen una competencia directa con los inmigrantes ${ }^{10}$.

Para obtener los beneficios deseados de la inmigración con alta calificación, en términos de innovación y productividad, es necesario que estos trabajadores sean empleados debidamente en sectores de alta tecnología. Estas ocupaciones podrían incluir gerentes y administradores, desarrolladores de software, enfermeras, contadores y auditores, analistas de sistemas informáticos, médicos, etc.

De acuerdo a la evidencia empírica, los inmigrantes se encuentran mayormente en los extremos dela distribución de capacidades de los trabajadores. Es decir, la mayoría de migrantes son de muy alta o muy baja calificación. En contraste, los trabajadores locales se concentran principalmente en el medio de dicha pirámide de distribución.

La especialización juega un rol importante en el aumento del producto y la productividad. Los trabajadores locales poseen las capacidades de comunicación y lenguaje más competitivos respecto a los inmigrantes. En consecuencia, los últimos requieren especializarme en sus ventajas más comparativas como trabajos manuales. Mientras que otros ciudadanos locales como los hombres de negocios, los inversionistas, incluso los terratenientes pueden verse beneficiados por mayores retornos económicos.

En caso que las capacidades difieran mucho del tipo de capacidades que poseen los trabajadores locales podría darse un mejor fenómeno de 
complementariedad entre estos dos tipos de trabajadores. De esta manera, ambos grupos son capaces de mostrar una mejor oferta laboral en favor del producto y la productividad. En contraste, el tamaño del mercado laboral podría verse reducido en caso exista un alto grado de sustitución entre los trabajadores locales y extranjeros; y por lo tanto, afectar negativamente el producto.

Por su parte, el incentivo a adquirir más capital, por parte de las empresas, aumentaría a medida que se cuenten con una dotación de trabajadores que cuenten con altos niveles de habilidades. Este capital podría incluir activos de capital y alta tecnología. Para obtener beneficios más inmediatos se requiere una economía intensiva en capital como en los casos de los países desarrollados que se han visto rápidamente beneficiados por la inmigración altamente calificada. De hecho, para el caso de Estados Unidos, los estudios señalan que los inmigrantes han mostrado una efectiva complementariedad con los trabajadores locales. En ese país se calculó que los inmigrantes reciben doble número de patentes que los nativos ${ }^{11}$.

Prácticamente, no habría dudas de un efecto positivo de la inmigración venezolana en la economía peruana, en un largo plazo. Sin embargo, se cuentan con problemas inmediatos, en el corto plazo, que deben resolverse. Como efecto inmediato, los inmigrantes compiten con los trabajadores locales que poseen las habilidades más bajas, ubicados en los puestos de trabajo menos calificados. Ello se debe a que los primeros, en muchos casos, carecen de acceso a la información y/o permisos legales laborales. Ello explica la gran preocupación y rechazo por parte de un importante sector; sobre todo de los fragmentos más pobre de la sociedad peruana.

Otro serio problema deriva de la existencia de un amplio sector informal que abarca alrededor de las tres cuartas partes de la economía peruana. En este contexto, es exiguo el aporte que pueden ofrecer los inmigrantes dado la carencia de capital físico u oportunidades para desempeñar sus habilidades y destrezas. Por el contrario, las economías que más se han visto beneficiadas por los flujos migratorios se han caracterizado por ser intensivas en capital, las cuales han absorbido velozmente los mayores niveles de fuerza laboral como ha sido el caso de EE.UU.

11 Ídem. 


\section{Situación de la inmigración en el Perú}

De acuerdo a los datos del Instituto Nacional de Estadística e Informática (INEI),la variación anual de la población en edad de trabajar (PET), es mayor para el caso de los inmigrantes que para la población peruana. Por ejemplo, en 2017 los primeros crecieron a una tasa 11.36\%; mientras que los segundos apenas en 1.55\%; es decir, cada año ingresan más los trabajadores extranjeros al mercado laboral respecto los peruanos. La mayoría de inmigrantes provienen de los países vecinos como Venezuela, Colombia, Argentina y Chile; mientras que en otras regiones se registran presencia de extranjeros residentes principalmente de Estados Unidos, España, Italia, China y Japón.

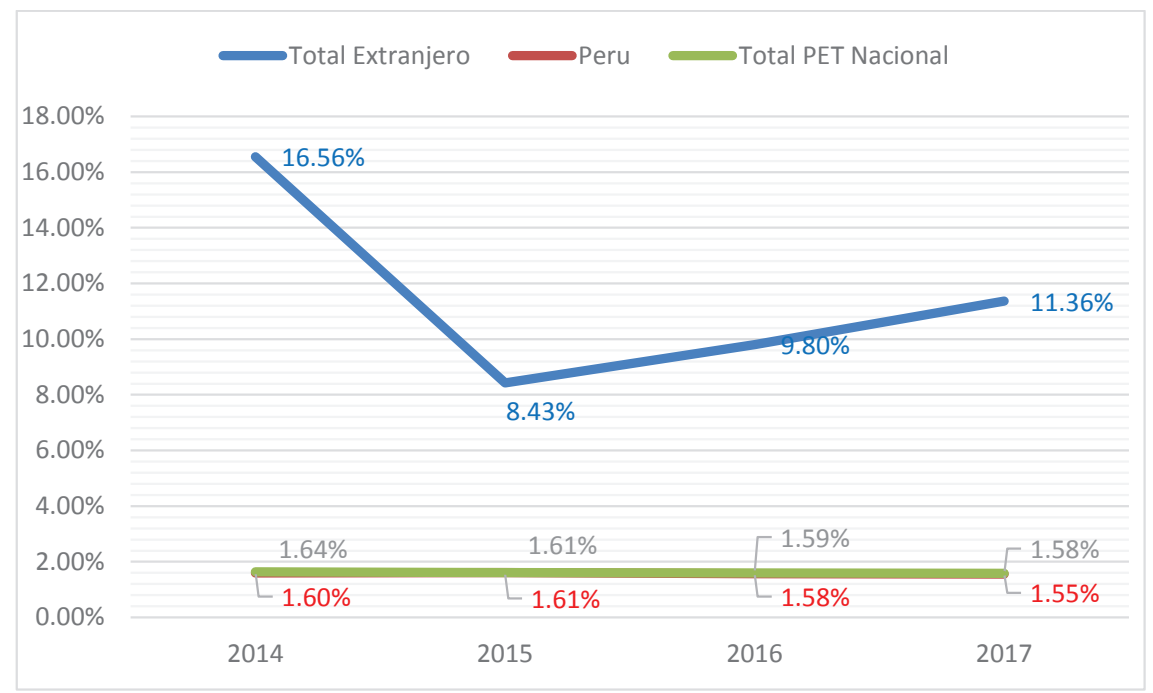

Gráfico 1. Variación anual de la PET inmigrante extranjera, PET peruana y total PET en el Perú

Fuente: INEI, ENAHO

La reciente llegada de ciudadanos venezolanos ha encendido debate público respecto los potenciales impactos de esta inmigración en la economía peruana. Los temores por parte de un gran número de peruanos, por la posible pérdida de sus empleos, está originando que muchos inmigrantes se encuentren expuestos a conductas de rechazo. En efecto, 
los inmigrantes tienden a causar un efecto sustitución si sus capacidades son similares a la de los trabajadores locales. Por el contrario, la economía se beneficia enormemente si se diera un efecto complementario. Por ejemplo, un país posee muchos administradores y requiere obreros, la inmigración de esto últimos brindaría un impacto positivo sin pérdidas de empleo.

En cualquier caso, la llegada de inmigrantes con un alto nivel educativo aumenta la productividad de la economía debido a que trabajadores con mayores capacidades elevarían la producción en términos de cantidad y calidad. En el caso de los inmigrantes venezolanos, se registra que la mayoría de ellos cuentan con grado superior de instrucción, lo cual no se estaría aprovechando dada las características de la economía peruana. En efecto, en el corto plazo no se observa una absorción clara o que los inmigrantes se estén insertando adecuadamente en el mercado laboral. Más bien estarían predominando los temores por riesgos de sustitución por parte de los trabajadores peruanos.

Los académicos discuten que porcentaje del total de la población económicamente activa (PEA) tendrían que ser la PEA inmigrante para calificar de significativo los posibles efectos de la inmigración. Como referencia, el estudio de David Card sobre la inmigración en Estados Unidos concluyó que el aumento de $10 \%$ en la PEA inmigrante respeto al total no tuvo ningún efecto negativo en la economía ni originaba caída en los salarios. Según las estadísticas del INEI, en los últimos años la participación de la PEA inmigrantes respecto al total de la PEA del mercado laboral peruano es menor al 1\%. De hecho, en los años 2016 y 2017 esta fue de aproximadamente $0.23 \%$ para cada año; lo que representa magros aumentos respecto a los años anteriores. Es importante destacar la PEA inmigrante, actualmente conformada principalmente ciudadanos venezolanos; pero también incluye otras nacionalidades como colombianos, argentinos, chilenos, etc.

Estos resultados insignificantes menores a 1\% difieren fuertemente con la percepción social de un fuerte ingreso de inmigrantes; la cual se explica por diversas razones. En primer lugar, el INEI categoriza como residentes a aquellas personas que se encuentran viviendo más de 30 días en la vivienda donde han sido encuestados. Se sabe que los nuevos 
inmigrantes venezolanos poseen un alto grado de movilidad y que estarían cambiando de domicilio en función de la búsqueda de oportunidades laborales; por tanto, un gran número de inmigrantes no estarían siendo considerados en las estadísticas. La PEA, por definición, elimina a aquellos ciudadanos menores de 14 años de edad, lo cual descarta otro número de inmigrantes del número total que reporta la Superintendencia Nacional de Migraciones. De otro lado, muchos ciudadanos venezolanos estarían manifestando no estar buscando empleo por las bajas condiciones que ofrece el mercado laboral peruano; por tanto, tampoco formarían parte de la PEA.

Cuadro 1. PEA inmigrante, peruana y total Perú; y participación de la PEA inmigrantes respecto a la PEA total

(en absoluto)

\begin{tabular}{|c|c|c|c|c|c|}
\hline & 2013 & 2014 & 2015 & 2016 & 2017 \\
\hline A. Inmigrantes & 25893 & 31879 & 34967 & 39474 & 39109 \\
\hline B. Peruanos & 16302951 & 16364498 & 16463171 & 16864207 & 17176632 \\
\hline C. Total Perú PEA & 16328844 & 16396377 & 16498138 & 16903681 & 17215741 \\
\hline $\begin{array}{l}\text { D. Participación de los } \\
\text { inmigrantes en la PEA } \\
\text { total (A/C) }\end{array}$ & $0,16 \%$ & $0,19 \%$ & $0,21 \%$ & $0,23 \%$ & $0,23 \%$ \\
\hline
\end{tabular}

Fuente: INEI - ENAHO

Es razonable afirmar que los inmigrantes que buscan empleo en el Perú estarían concentrados en las principales ciudades; por ende, sería más correcto calcular la participación de la PEA inmigrante respecto al total por ciudades. De este ejercicio se encontró que, Tacna, Limay Callao son las tres principales ciudades con los mayores ratios; no obstante, en estos tres casos se sigue contando con ratios menores al $1 \%$. Cabe mencionar que estas ciudades han sido catalogadas como las ciudades de transito de los migrantes venezolanos de acuerdo a estudios de la Organización Internacional de Migraciones (OIM); otra ciudad es tumbes. A través de estas ciudades los ciudadanos venezolanos llegan o salen de Perú principalmente por vía aérea, por bus nacional y bus internacional. 
Pensamiento Crítico Vol. 23. N²

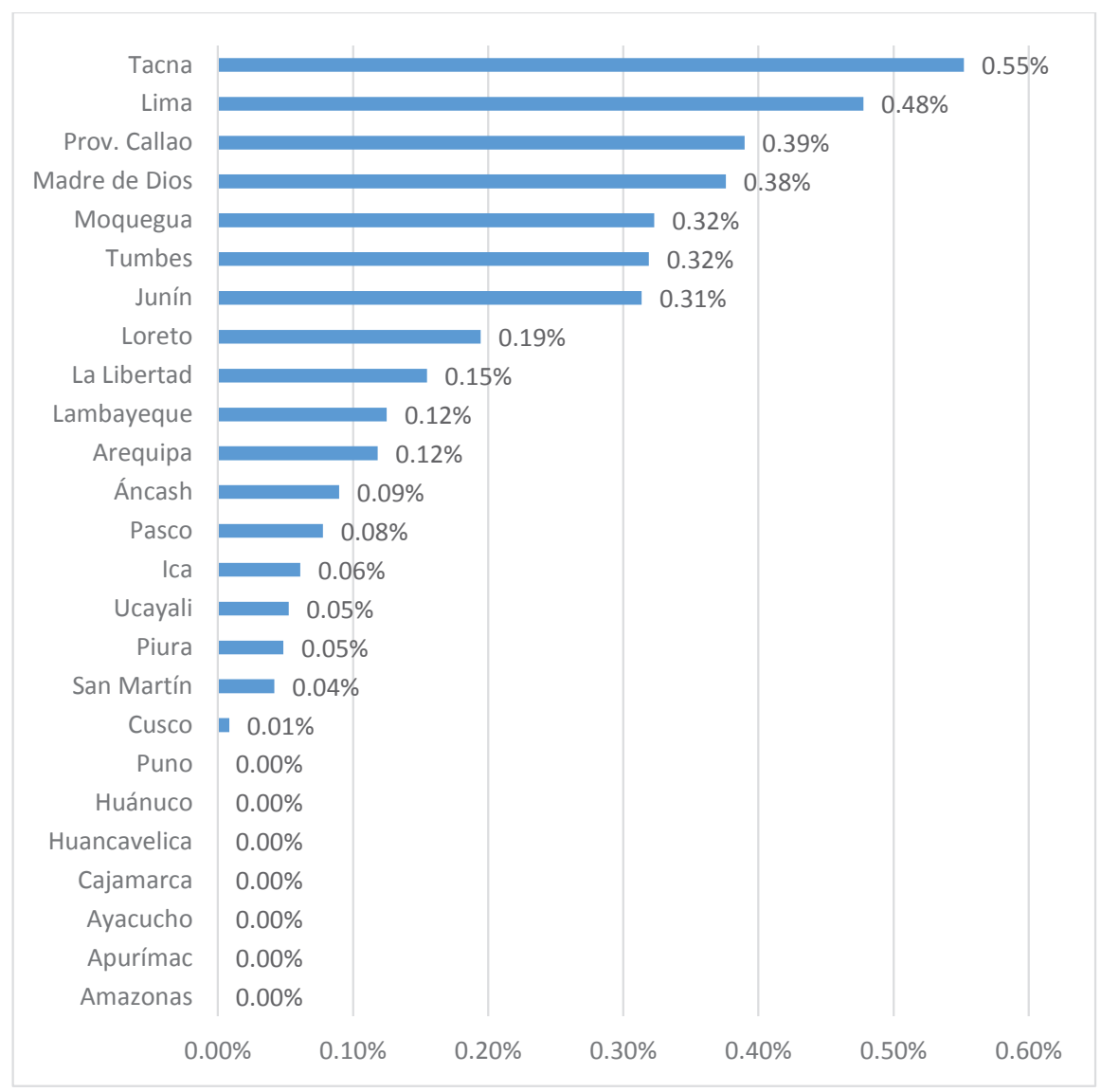

Gráfico 2. Participación de la PEA inmigrantes respecto a la PEA total, según departamento, 2017

Fuente: INEI, ENAHO

Como ya se mencionó, un importante número de inmigrantes venezolanos en edad de trabajar, habrían dejado de buscar empleo debido a las bajas condiciones que ofrece el mercado laboral peruano. En consecuencia, al momento de ser encuestados por el INEI, quedan registrados en la condición de actividad de 'inactivos'; es decir, entre aquellos que no tienen empleo, pero tampoco lo están buscando ni tienen interés en hacerlo. Es importante destacar que entre los años 2016 y 2017 los inmigrantes inactivos en Perú aumentaron considerablemente en un 30\%, lo cual contrasta 
ampliamente con los no-inmigrantes (trabajadores peruanos) que solo aumentaron en un 1\%, aumento similar al promedio total del mercado laboral en el Perú. El fuerte aumento de inmigrantes que se declaran como inactivos también redujo el ratio PEA inmigrante respecto a la PEA total. Sin embargo, es probable que algunos de estos inmigrantes inactivos realicen eventuales actividades laborales, sobretodo en la economía informal.

De otro lado, los inmigrantes desocupados aumentaron en un 107\%; contrario al número de desocupados peruanos que cayó en un 1\%.Con respecto a los trabajadores ocupados, en el año 2017 hay menor número de inmigrantes ocupados respecto al año anterior, cayendo en un 11\%; mientras que el número de trabajadores peruanos ocupados aumentó en el orden del 2\%. Esto sería un indicador de la desfavorable situación que recientemente afrontan los inmigrantes en Perú. Es importante destacar que economías como Estados Unidos o Alemania se beneficiaron rápidamente de la inmigración debido a que a son países intensivos en capital. Por tanto, la nueva fuerza laboral requiere de economías intensivas en capital para alcanzar una complementación en favor del crecimiento económico.

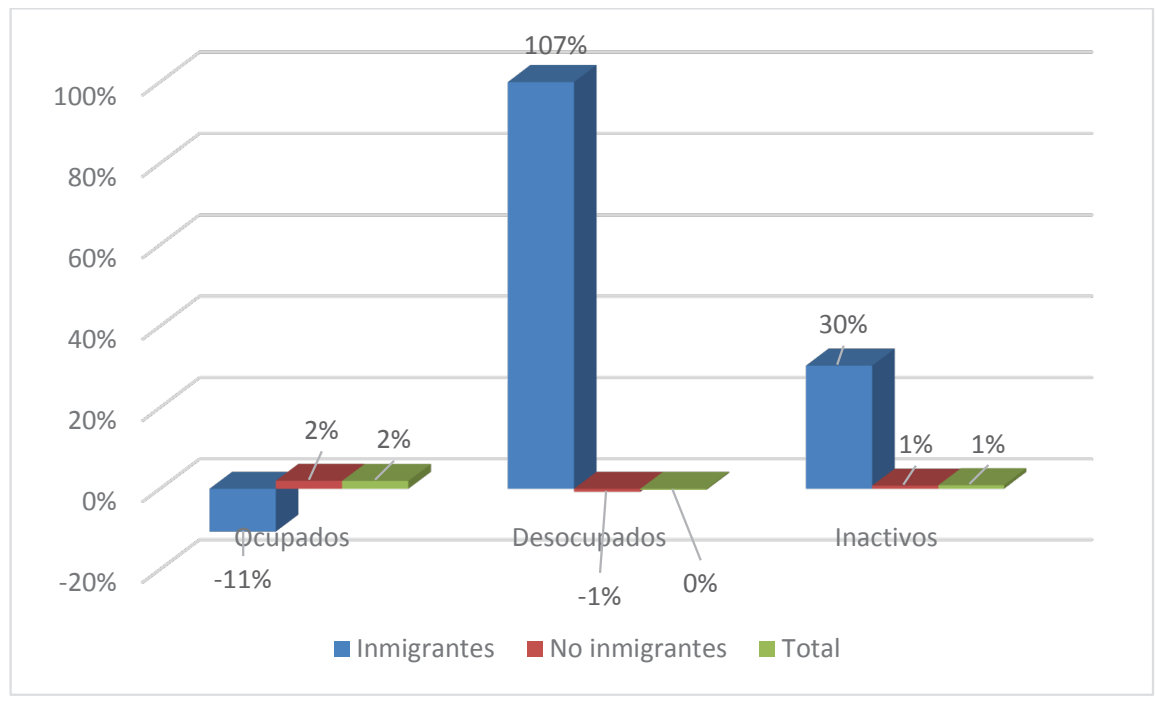

Gráfico 3. Variación 2016-2017 de los inmigrantes, peruanos y total por condición de actividad

Fuente: INEI, ENAHO 
Los reportes oficiales publican cifras de más de 300 mil venezolanos que han ingresado al Perú y con recientes casos de 5000 venezolanos que ingresan diario solo por la frontera con Tumbes. Estas cifras perderían relevancia si se considera que alrededor de la cuarta parte de estos nuevos inmigrantes venezolanos tienen como propósito hacer tránsito a otros países como Argentina y Chile; y en menor medida otros destinos como Europa o Estados Unidos. Según la segunda ronda del estudio de inmigración venezolana de la OIM, un $23 \%$ de inmigrantes venezolanos transita por el Perú hacia otros países; de los cuales, principalmente, un 19\% se va a Chile y un 3\% a Argentina.

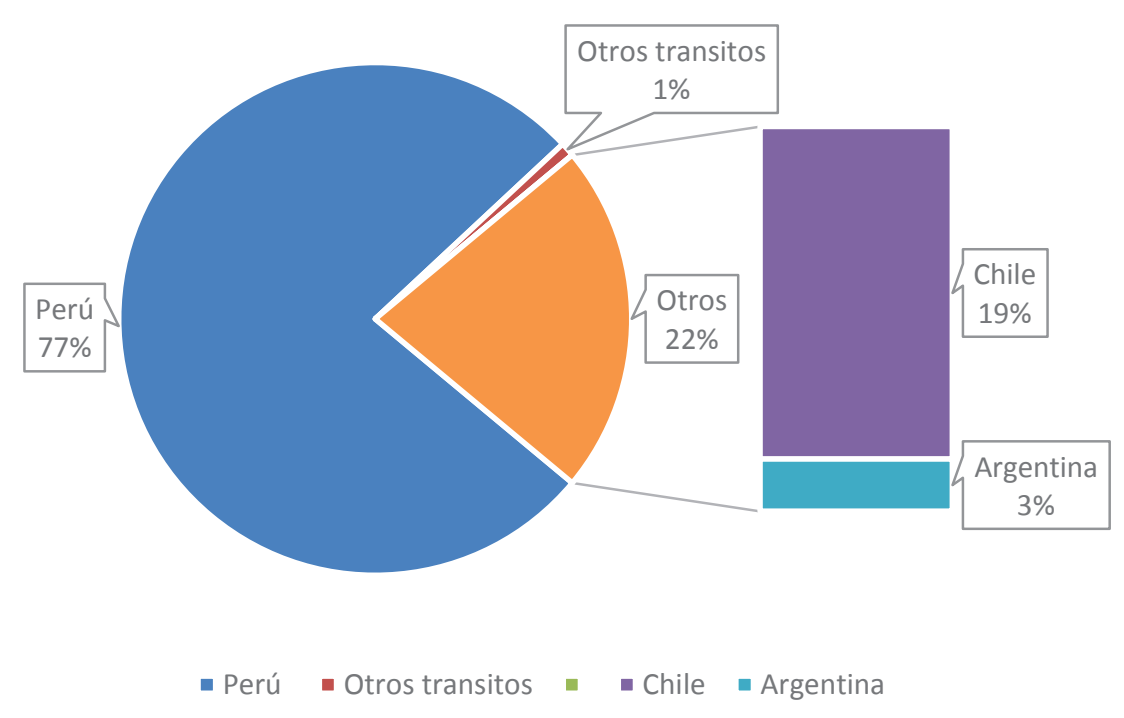

Gráfico 4. Destino de los inmigrantes venezolanos que llegan al Perú Fuente: OIM-Monitoreo de Flujo de Migración Venezolana, Ronda 2

Las razones de tránsito hacia terceros países no solo se deben a mayores oportunidades labores, sino los inmigrantes venezolanos también buscan reunirse con otros familiares ya establecidos en los países de destinos finales. Es posible que estos inmigrantes en tránsito estén realizando actividades laborales temporales dentro del mercado informal en el Perú, sin los permisos correspondientes como el PTP (permiso temporal de permanencia). Ello explicaría la sobredimensionada presencia de 
ciudadanos venezolanos que no aparecen en las estadísticas; sobre todo por hacerse más visibles ante la sociedad en actividades informales. Ello sería un agravante de la carencia de una correcta política migratoria con la falencia del debido control y regulación de ciudadanos extranjeros que incluso habría permitido el ingreso de algunos extranjeros que incurren en prácticas delictivas, lo cual afecta la imagen del inmigrante en general.

\section{Efecto de la inmigración en los salarios}

En el 2017 se observa una desaceleración en la tendencia creciente que venía experimentando el salario promedio del mercado laboral en el Perú. En efecto, en el siguiente grafico se muestra un quiebre en el último año en la evolución del salario. Se contaba con una tendencia distinta hasta el 2016, cuando se llegó a un salario promedio de S/ 1233 , partiendo de un valor de S/ 1065 en el 2013. El aumento del último año corresponde a apenas $\mathrm{S} / .4$, lo que representa un crecimiento cercano a $0 \%$.

Por su parte, la teoría predice efectos negativos en el salario en el corto plazo producto de olas inmigratorias. Sin embargo, como venimos sosteniendo, estos efectos se darían sobretodo en el corto plazo, afectando principalmente a los sectores más vulnerables, quienes compiten más directamente con los nuevos inmigrantes. Ante ello, el gobierno debe implementar políticas públicas para capacitar a los trabajadores peruanos que cuentan con menos calificaciones y que son más afectados por la inmigración, a través del Ministerio de Trabajo y Promoción del Empleo (MTPE). Ello no solo ayudaría a solucionar el problema de ingreso de estos sectores más vulnerables del país; sino también aumentará el promedio de la productividad a nivel nacional.

Un análisis más detallado de los salarios describe una tendencia negativa en la evolución del salario promedio de los trabajadores inmigrantes en el Perú; mientras que el promedio salarial de los trabajadores peruanos mantiene una tendencia positiva. Es importante destacar que tradicional el promedio salarial de los primeros ha sido mayor que los segundos. Sin embargo, estas tendencias descritas están haciendo que ambos promedios tiendan a converger. Es importante destacar que dentro del grupo de trabajadores inmigrantes, los venezolanos son quienes vienen sufriendo la mayor caída en su salario. 


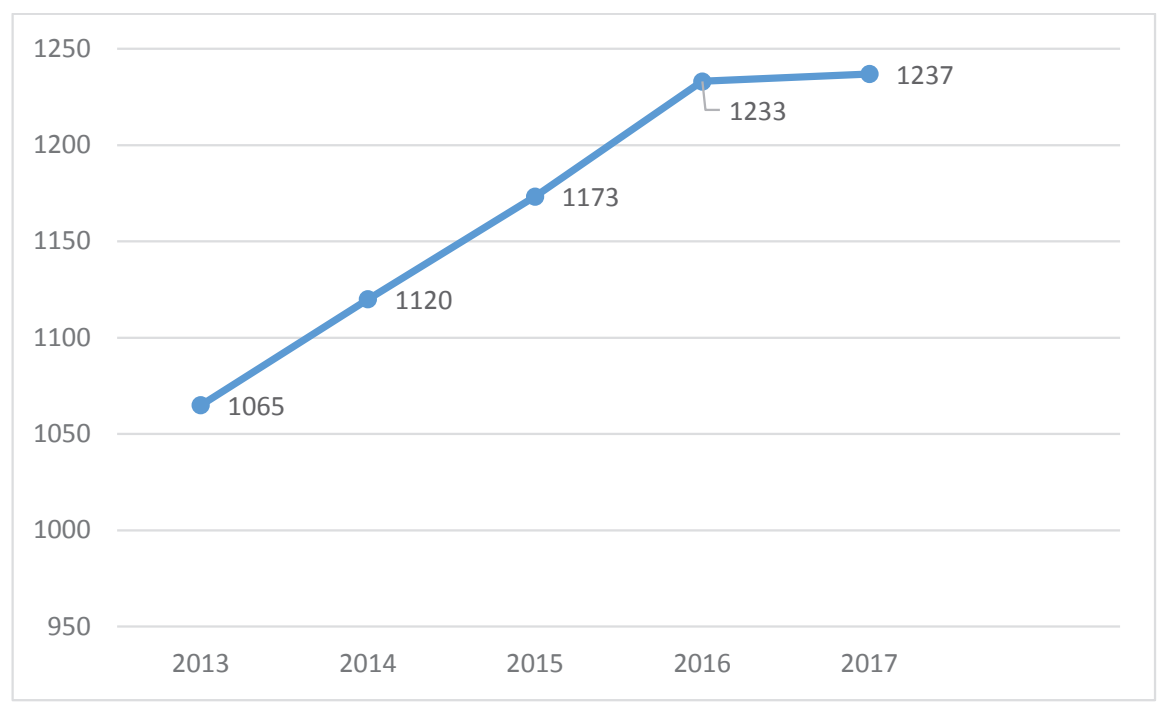

Gráfico 5. Evolución del promedio de salarios del total del mercado laboral en el Perú (en Soles)

Fuente: INEI-ENAHO

Lo anterior sugiere un efecto sustitución entre los nuevos y antiguos inmigrantes de la misma nacionalidad; es decir, los inmigrantes venezolanos que ya laboran en el mercado laboral sufrirían los mayores riesgos de desempleo o desplazo de su puesto de trabajo por la llegada de nuevos de inmigrantes de su misma nacionalidad. En efecto, es razonable afirmar que personas del mismo país posean más similitud en cuanto a sus capacidades y formación educativa; respecto a personas de otros países.

\section{Los inmigrantes en el mercado informal en el Perú}

La informalidad de la economía peruana abarca casi las tres cuartas partes de esta. Según cifras oficiales del INEI en el 2017 la informalidad alcanzó un porcentaje del $73 \%$. Es importante destacar que, similarmente a la evolución del salario promedio, en el último año hubo un aumento en la informalidad revertiendo la tendencia favorable que se venía acumulando hasta el 2016 cuando esta venía cayendo ligeramente desde 75\% hasta el 72\%. De acuerdo con algunas críticas, el reciente aumento de la informalidad es atribuido, en parte, a los nuevos inmigrantes venezolanos 
que se observan en las vías públicas realizando actividades relacionadas al comercio ambulatorio.

Según las cifras oficiales, los trabajadores inmigrantes en el mercado laboral peruano presentan niveles de informalidad menores respecto al total nacional; sin embargo, ambos siguen similar trayectoria. En efecto, del 2013 al 2016 la informalidad del grupo de los inmigrantes venía cayendo desde un porcentaje de 52\% hasta un $45 \%$. No obstante, en el 2017 esta tendencia se revirtió aumentando 3 puntos porcentuales hasta llega a una cifra de $48 \%$. Este comportamiento refuerza la relación entre la reciente migración en el Perú y el aumento de la informalidad.

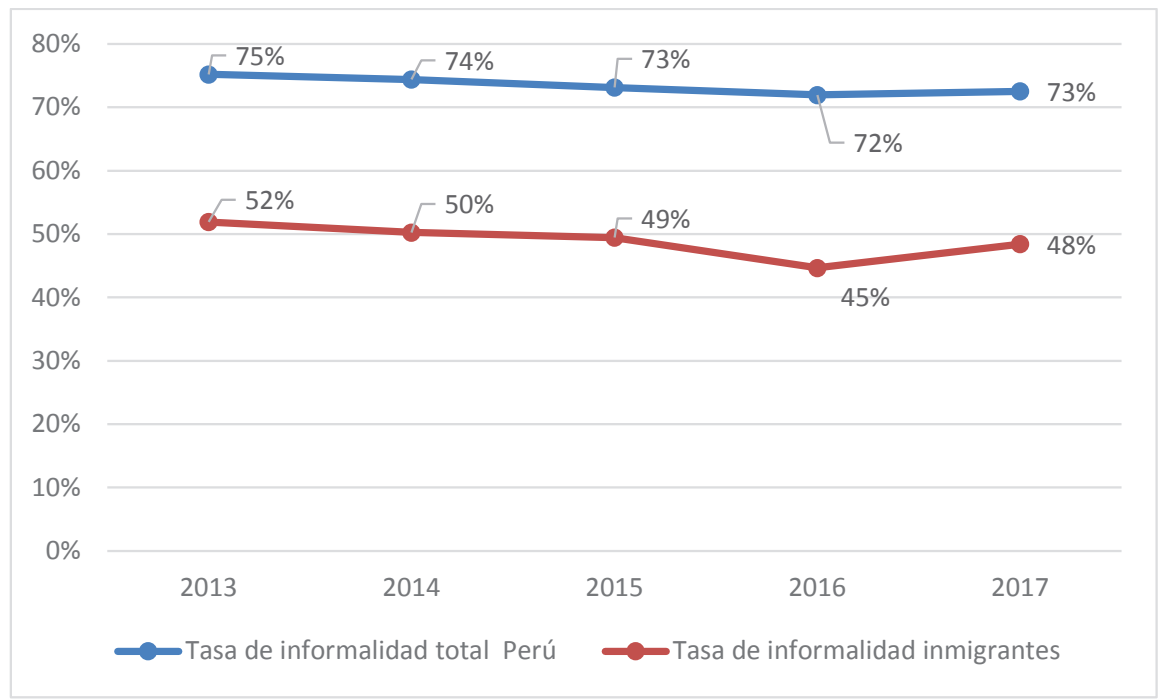

Gráfico 6. Comparación ente la informalidad total y de inmigrantes en el mercado laboral en el Perú

Fuente: INEI-ENAHO

El trabajo informal de los inmigrantes es causado en muchos casos por irregularidades en su ingreso y/o faltas de permiso para trabajar dentro del país de destino. De hecho, las instituciones oficiales en Perú registran que la mayoría de inmigrantes venezolanos ingresan en calidad de turistas y mientras buscan cambiar su régimen y obtener el PTP, ellos vienen realizando actividades informales. Se estima que solo una minoría de inmigrantes venezolanos que laboran en Perú posee el PTP. 
Según la metodología del INEI y del MTPE para poder ser considerado formal en Perú, básicamente se requiere que el trabajador cuentan con el registro único del contribuyente RUC; asimismo que goce de seguro de salud. Los trabajadores que no cuentan con estos requisitos son considerados informales. El mayor trabajo informal se concentra en las empresas pequeñas o independientes. Las instituciones peruanas tienen una larga data de fracasos, para poder solucionar el problema de la informalidad en el país. En este marco, los nuevos inmigrantes se ven incentivados a aceptar empleos informales o deciden trabajar como independientes. El sector informal es un indicador de falta de servicios públicos, infraestructura y capital físico. Por tanto, los trabajadores tanto nacionales como extranjeros que se inserten en este mercado terminan ofreciendo baja productividad que no les permite obtener los ingresos suficientes para satisfacer sus necesidades.

Una mejor política migratoria en Perú debe incluir shocks de inversión para que las empresas puedan contratar la nueva masa laboral que ingresa al país; sobre todo aprovechar aquellas que cuenta con un alto nivel educativo o capital humano. De esta manera, las empresas podrán aumentar sus niveles de producción sin afectar los salarios de los trabajadores, gracias al aumento de la demanda laboral. Ello haría que la economía alcance más aceleradamente los efectos positivos de la inmigración de largo plazo que se analizó en la figura 1.

\section{Los inmigrantes en el mercado formal en Perú}

La Planilla Electrónica es la información del MTPE recogida básicamente de empresas formales. Para el presente artículo se ha solicitado a esta institución el permiso correspondiente para la publicación de algunas de sus estadísticas importantes para reformar nuestras proposiciones. De esta manera, para obtener conclusiones del impacto de los inmigrantes en el mercado laboral en el Perú se hará uso de esta fuente de información, la cual está disponible en periodos mensuales para diferentes características de los trabajadores.

El siguiente grafico muestra un aumento exponencial de los trabajadores venezolanos respecto a los inmigrantes de las demás nacionalidades, quienes provienen principalmente de Colombia, España, China, 
Argentina y Chile. En efecto, el número de trabajadores venezolanos aumentó desde un nivel ligeramente superior de 1000 trabajadores en enero del 2016 hasta cantidades cercanas a 6000 trabajadores venezolanos a noviembre del 2017. Se espera que esta tendencia continúe para los próximos meses debido a continua crisis económica, social y política que persiste en Venezuela más las facilidades aprobadas por parte del gobierno del Perú.

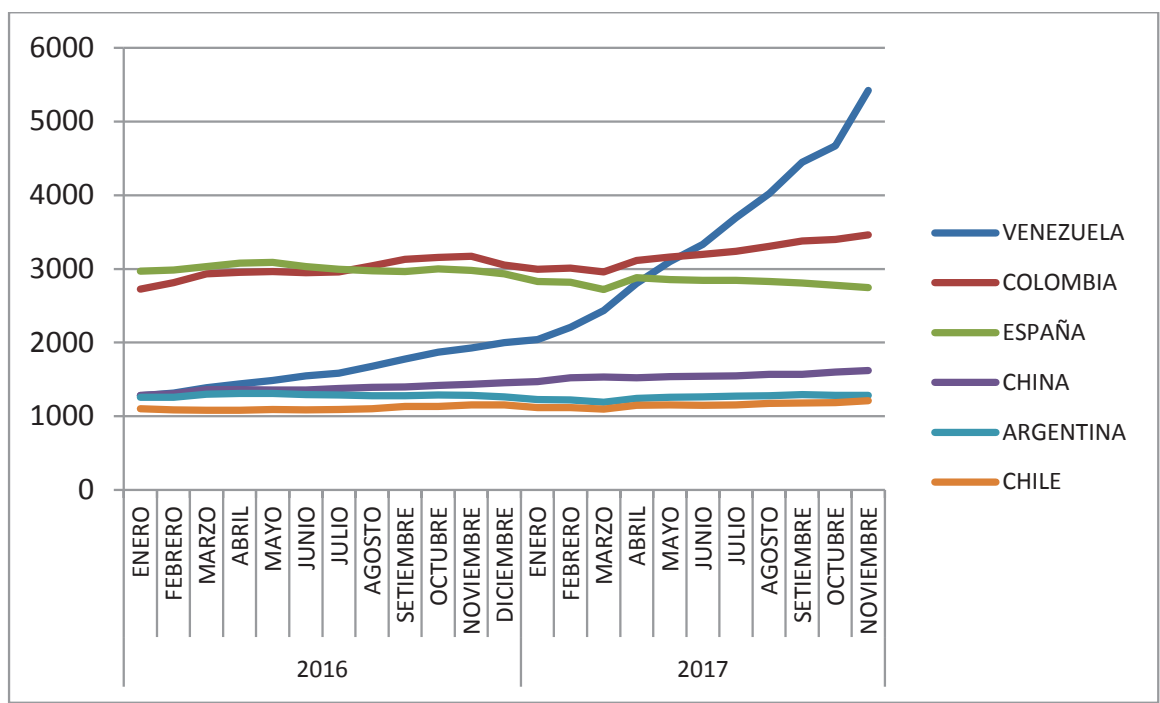

Gráfico 7. Evolución mensual de los principales inmigrantes dentro del mercado laboral formal en el Perú

Fuente: Planilla Electrónica - MTPE

Como había sido propuesto anteriormente, la inmigración ha hecho que se genere más competencia en las ocupaciones donde se requiere menores calificaciones o grado de instrucción por parte de los trabajadores. La información de la Planilla Electrónica permite identificar las ocupaciones en la que más aumentó el número de venezolanos. Por ejemplo, las tres relevantes son: ayudante de camión con un incremento superior al 5000\%, mozos y trabajadores de casino con crecimientos cercanos al $200 \%$, etc. Es importante destacar que estas ocupaciones son las primeras en ranking de una lista detallada de cientos de ocupaciones registradas en la Planilla Electrónica. 


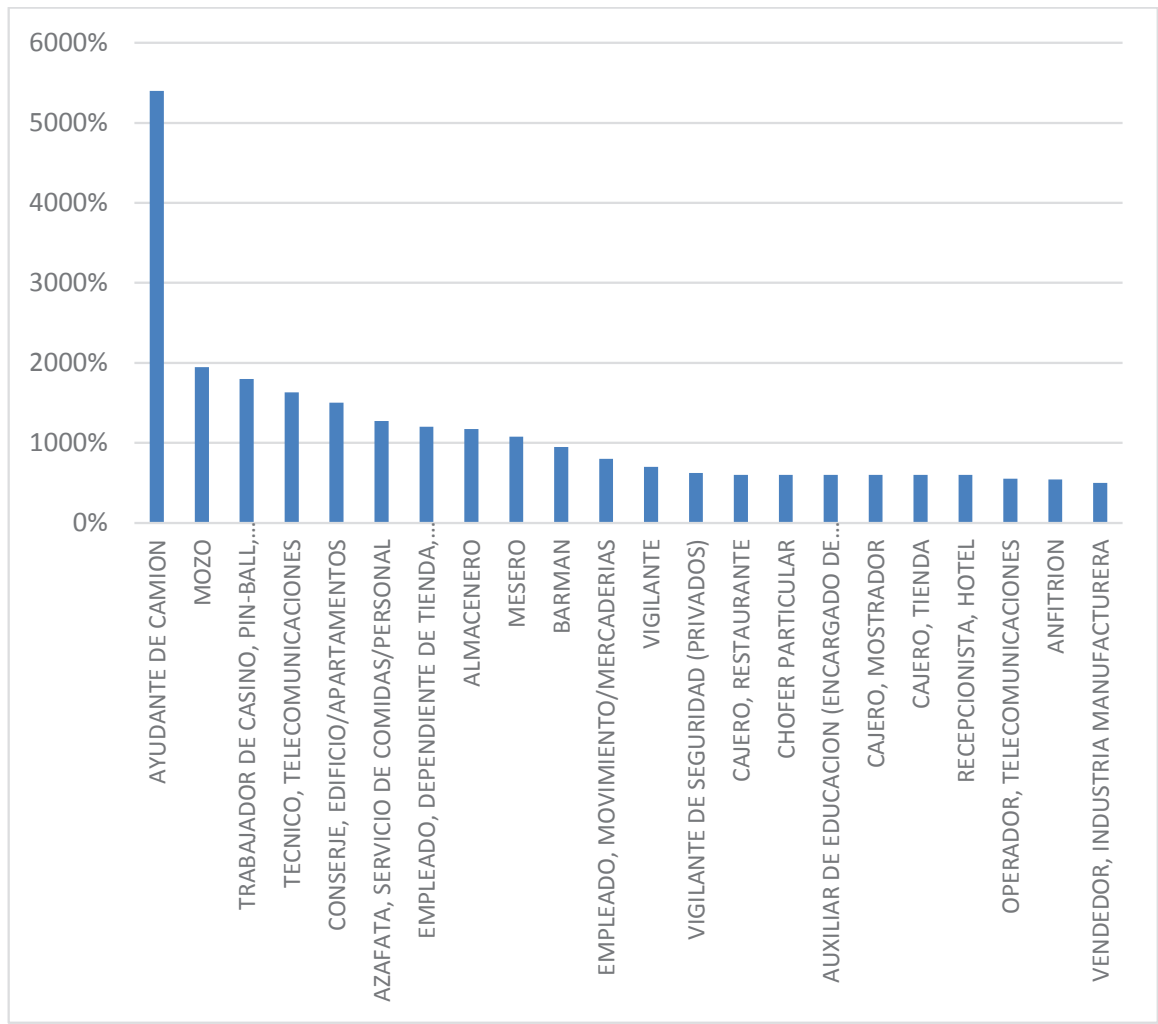

Gráfico 8. Principales variaciones en las ocupaciones de los inmigrantes venezolanos en el mercado laboral formal del Perú entre diciembre del 2016 y noviembre del 2017

Fuente: Planilla Electrónica - MTPE

\section{Conclusiones}

Como hemos venido sosteniendo, la inmigración es beneficiosa para todas las economías en el largo plazo. Sin embargo, los problemas analizados en el corto plazo requieren de la implementación de políticas públicas para afrontarlos. Es importante destacar que las características del mercado laboral en el Perú, tales como un alto nivel de informalidad o las carencias de capital físico e infraestructura pública harían que estos problemas perduren por muchos años. Un caso contrario ha ocurrido en países como Estados Unidos donde los efectos positivos han sido prácticamente inmediatos. 
Entre las principales recomendaciones de políticas públicas propuestas destacan: (i)una mejora de la política migratoria mediante una mayor regulación y control de ciudadanos extranjeros, para evitar prácticas delictivas por parte de los extranjeros, que atenten contra la seguridad interna; asimismo, legalizar a los inmigrantes para evitar que se inserten en la economía informal; (ii) la implementación de shocks de inversión para que las empresas privadas puedan absorber la nueva fuerza laboral, sobre todo aquella que cuenta con alto nivel de capital humano, de esta manera, aumentar la producción nacional y contribuir al crecimiento económico; (iii) Capacitar y generar oportunidades de empleo, dirigido por el MPTE, a favor de los trabajadores peruanos que laboran en los sectores más vulnerables y con menores capacidades, quienes son los más afectados por la mayor competencia de nuevos inmigrantes; esto a su vez, aumentaría la productividad nacional. 


\section{Anexos}

\section{Coeficiente de variación del total de inmigrantes}

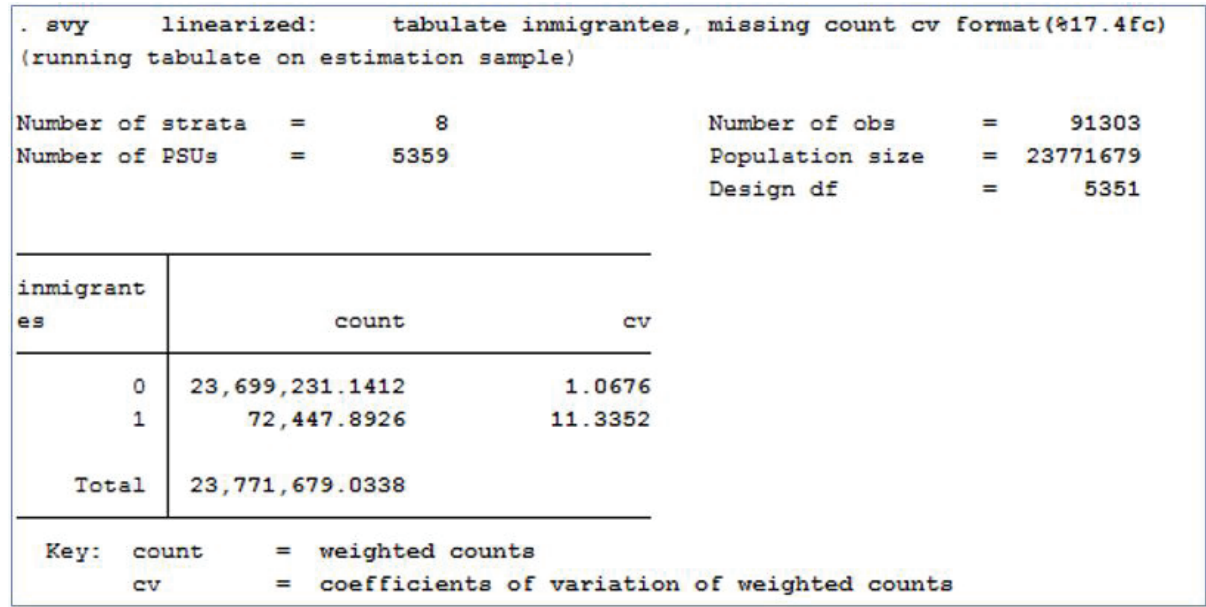

\section{Coeficiente de variación de los inmigrantes venezolanos}

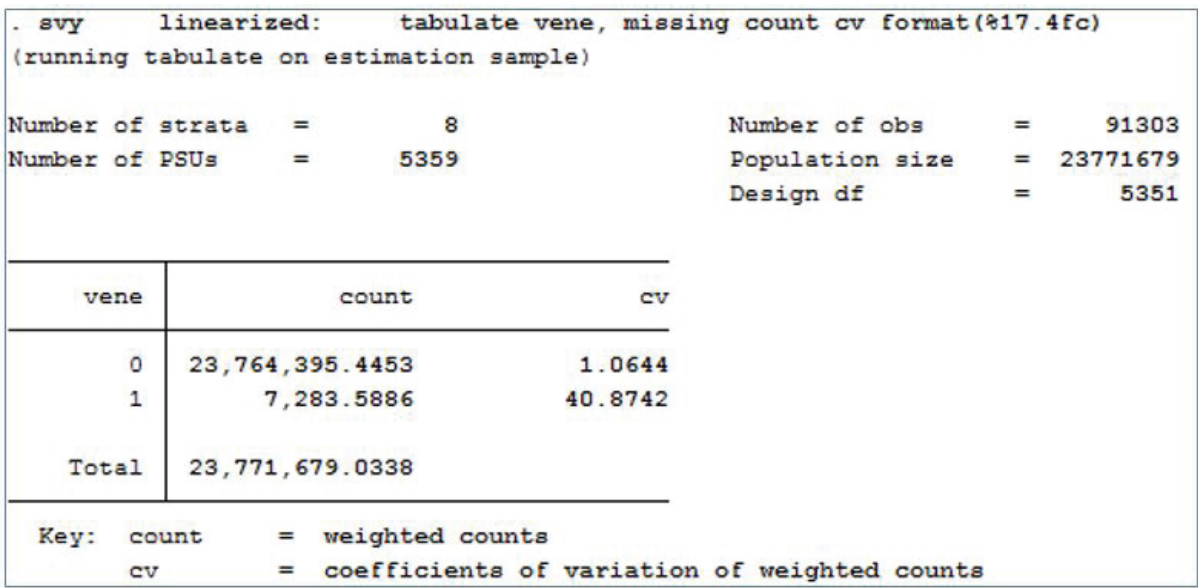




\section{Referencias Bibliográficas}

Raphael, S.; Lucas R. 2007.THE EFFECTS OF LABOR MARKET COMPETITION WITH IMMIGRANTS ON THE WAGES AND EMPLOYMENT OF NATIVES What Does Existing Research Tell Us? Du Bois Review, 4:2 (2007) 413-432. (C) 2007 W. E. B. Du Bois Institute for African and African American Research.

Somerville, W.; Sumption, M. 2009. Immigration and Labor Market: Theory, evidence and policies. Migration Policy Institute.

Smith, J. P. 2005. Immigrants and the Labor Market. WORKING PAPER, WR-321 November.

Docquier, F., Özden Ç., Peri G. 2011. The Labor Market Effects of Immigration and Emigration in OECD Countries. ForschungsinstitutzurZukunft der Arbeit Institute for the Study of LaborIZA DP No. 6258.

Beyer, R. C. M. 2016.The Labor Market Performance of Immigrants in Germany. WP/16/6 IMF, Working Paper, European Department.

Kaneta, K. 1999. Immigration and its Effects on U.S. Labor Markets.The Park Place Economist / vol. Viii.

Bosh, M., Farré L. 2013. Immigration and the Informal Labor Market.IZA DP No. 7843.

Gauten City-Region Observatory. 2014. URBAN INFORMALITY AND MIGRANT ENTREPRENEURSHIP IN SOUTHERN AFRICAN CITIES. 10-11 February 2014, Cape Town, South Africa Conference Report.

Flórez, C. E. 2003. Migration and the Urban Informal Sector in Colombia. Universidad de Los Andes Colombia, Paper prepared for Conference on African Migration in Comparative Perspective, Johannesburg, South Africa, 4-7 June, 2003.

Espíndola, A., Silveira, J., Penna, T. 2006. A Harris-Todaro Agent-Based Model to RuralUrban Migration. Brazilian Journal of Physics, vol. 36, no. 3A, September, 2006.

Dustmann, C., Hatton, T. and Preston, I. 2005.THE LABOUR MARKET EFFECTS OF IMMIGRATION.The Economic Journal, 115 (November), F297-F299._ Royal Economic Society 2005. Published by BlackwellPublishing, 9600 Garsington Road, Oxford OX4 2DQ, UK and 350 Main Street, Malden, MA 02148, USA.

Somerville, W. and M. Sumption.2009. Immigration and the labour market: Theory, evidence and policy. Equality and Human Rights Commission.

Orrenius, P. \& Zavodny, M. 2013.Immigrants in the US Labor Market. Federal Reserve Bank of Dallas Research Department Working Paper 1306, September 2013.

Sharma, A. N., Sood, D. 2008. Migration and Informality. Institute for Human Development, New Delhi. 